ISSN 0103-5150

Fisioter. Mov., Curitiba, v. 26, n. 3, p. 491-502, jul./set. 2013

Licenciado sob uma Licença Creative Commons

\title{
Comparação de diferentes testes funcionais de membros inferiores em pacientes com doença pulmonar obstrutiva crônica: há concordância entre eles? ${ }^{1}$
}

\author{
Comparison of different functional tests of lower \\ limbs in patients with chronic obstructive pulmonary \\ disease: there is concordance between them?
}

\section{Bruna Varanda Pessoa ${ }^{[a]}$, Mauricio Jamami ${ }^{[b]}$, Renata Pedrolongo Basso ${ }^{[c]}$, Eloisa Maria Gatti Regueiro ${ }^{[c]}$, Antônio Delfino de Oliveira Jr. ${ }^{[\mathrm{d}]}$, Valéria Amorim Pires Di Lorenzo ${ }^{[\mathrm{e}]}$}

[a] Doutora em Fisioterapia pela Universidade Federal de São Carlos (UFSCar), São Carlos, SP - Brasil, e-mail: brunavpessoa@gmail.com

[b] Doutor em Fisioterapia pela Universidade Federal de São Carlos (UFSCar), professor associado do curso de Graduação em Fisioterapia e Programa de Pós-Graduação em Fisioterapia da Universidade Federal de São Carlos (UFSCar), São Carlos, SP - Brasil, e-mail: jamami@ufscar.br

[c] Doutora em Fisioterapia pela Universidade Federal de São Carlos (UFSCar), São Carlos, SP - Brasil, e-mails: renata.fisio@gmail.com, eloregueiro@yahoo.com.br

[d] Médico pneumologista da Santa Casa de Misericórdia de São Carlos, SP - Brasil, e-mail: adojr@terra.com.br

[e] Doutora em Fisioterapia pela Universidade Federal de São Carlos (UFSCar), professora associada do curso de Graduação em Fisioterapia e Programa de Pós-Graduação em Fisioterapia da Universidade Federal de São Carlos (UFSCar), São Carlos, SP - Brasil, e-mail: vallorenzo@ufscar.br

Resumo

Introdução: Pacientes com Doença Pulmonar Obstrutiva Crônica (DPOC) apresentam diminuição da capacidade funcional, tornando imprescindível a sua avaliação por meio de testes físicos funcionais. Objetivos: Comparar, em pacientes com DPOC, saturação periférica de oxigênio $\left(\mathrm{SpO}_{2}\right)$, dispneia e fadiga de membros inferiores (MMII) nos testes de caminhada de seis minutos em corredor (TC6) e em pista oval (TC6Po), do degrau de seis minutos (TD6) e de sentar-e-levantar da cadeira de dois minutos (TSL).

\footnotetext{
1 Trabalho realizado no Laboratório de Espirometria e Unidade Especial de Fisioterapia Respiratória, Departamento de Fisioterapia Universidade Federal de São Carlos, São Carlos, SP - Brasil. Número de identificação no Registro de Ensaios Clínicos: NCT00660738.
} 
Além disso, pretende-se verificar se há correlação e concordância dessas variáveis entre o TC6 e os demais testes. Materiais e métodos: Foram avaliados 11 pacientes com DPOC ( $71 \pm 8$ anos, $\mathrm{VEF}_{1}<80 \%$ previsto) por meio dos testes. Resultados: Na análise intertestes, não se observaram diferenças significativas nos deltas da $\mathrm{SpO}_{2}$, dispneia ( $\Delta$ dispneia) e fadiga de MMII ( $\Delta$ fadigaMMII). Constatou-se correlação significante, mas não concordância entre os menores valores da $\mathrm{SpO}_{2}$ no TC6 com os menores valores da $\mathrm{SpO}_{2}$ nos TC6Po, TD6 e TSL, sendo a média da diferença entre as médias com \pm 1,96 desvio padrão: $0,8 \pm 3,5 ;-1,9 \pm 3,5$ e -2,5 \pm 4,6 , respectivamente; entre $\Delta$ dispneia e $\Delta$ fadigaMMII no TC6 com $\Delta$ dispneia e $\Delta$ fadigaMMII no TC6Po: 0,0 \pm 1,0 e $0,4 \pm 0,8$, respectivamente; e do $\Delta$ fadigaMMII no TC6 com $\Delta$ fadigaMMII no TSL: $0,1 \pm 1,1$. Conclusão: Os testes funcionais produziram respostas de oxigenação e perceptuais de esforço semelhantes em magnitude. Entretanto, de acordo com as variáveis analisadas, não foi encontrada concordância entre o TC6 com os demais testes, pelas grandes variações dos limites de concordância e grande variação interindividual, assim o TC6Po, TD6 e TSL não substitui o TC6.

Palavras-chave: Doença Pulmonar Obstrutiva Crônica. Teste de esforço. Dispneia. Fadiga muscular. Tolerância ao exercício.

\section{Abstract}

Introduction: Patients with Chronic Obstructive Pulmonary Disease (COPD) have reduced functional capacity, becoming indispensable to their evaluation through functional physical tests. Objective: To compare the peripheral oxygen saturation $\left(\mathrm{SpO}_{2}\right)$, dyspnea and fatigue of the lower limbs ( $\left.L L\right)$, between the six-minute walk test in hallway (6MWT) and the of oval track (6MWToT), the six-minute step test (6MST) and the sit-to-stand test (STST), in patients with COPD; also verifying correlate and concordance of these variables between 6MWT and other tests. Materiais e methods: Ten patients with COPD (72 \pm 7 years, FEV $<80 \%$ predicted) were assessed using the functional tests. Results: In the inter-tests analysis, there was no significant difference in $\mathrm{SpO}_{2}$ and in the change in dyspnea (Adyspnea) and fatigue in the lower-limbs ( $\mathrm{f}$ fatigueLL) between rest and exercise of peak ( $\Delta$ ). Significant correlation were observed, but no concordance between the lowest values of $\mathrm{SpO}_{2}$ in the 6MWT with lowest values of $\mathrm{SpO}_{2}$ in 6MWToT, 6MST and STST, and the mean of difference between the mean with standard deviation $\pm 1.96: 0.8 \pm 3.5 \%,-1.9 \pm 3.5 \%$ and $-2.5 \pm 4.6 \%$, respectively; and between $\triangle$ dyspnea and $\triangle$ fatigueLL in the $6 M W T$ with the $\triangle$ dyspnea and $\triangle$ fatigueLL in the 6MWTOT: $0.0 \pm 1.0$ and $0.4 \pm 0.8$, respectively; and $\triangle$ fatigueLL in the 6MWT with $\triangle$ fatigueLL in the STST: 0.1 \pm 1.1. Conclusion: The functional tests assess oxygenation and perceived effort responses similar in magnitude. However, according to the variables analyzed, weren't found correlation between the 6MWT with the 6MWToT, 6MST and STST, by the large variations in the limits of agreement and large interindividual variation, thereby the 6MWToT, 6MST and STST and doesn't replace the 6MWT.

Keywords: Chronic Obstructive Pulmonary Disease. Exercise test. Dyspnea. Muscle fatigue. Exercise tolerance..

\section{Introdução}

A Doença Pulmonar Obstrutiva Crônica (DPOC) é caracterizada por perda de peso significativa, redução da força e endurance dos músculos respiratórios, dos membros inferiores (MMII) e superiores (MMSS), causando diminuição da capacidade funcional e intolerância aos esforços (1).

A diminuição da capacidade de exercício deve-se à interação de múltiplos fatores, dentre eles, a limitação ventilatória, uma característica patofisiológica importante, responsável pela hipoxemia e dessaturação, principalmente nos estágios mais avançados da doença, além do sintoma de dispneia, que piora durante o exercício devido às inadequações da relação ventilação/perfusão (2), à fraqueza dos músculos inspiratórios, e à hiperinsuflação pulmonar (3). Outro fator limitante é a disfunção muscular periférica, em que há predomínio do metabolismo anaeróbico, com maior lactacidemia, contribuindo para a hipoxemia 
e para a fadiga muscular $(1,2)$. Isso resulta em dispneia, fraqueza muscular e descondicionamento físico, e por consequência, uma espécie de círculo vicioso ou espiral negativo, que gera importantes limitações funcionais em pacientes com DPOC (1-4).

Dessa maneira, torna-se imprescindível avaliar a capacidade funcional dos pacientes com DPOC por meio de diferentes testes físicos funcionais. Esses testes são métodos simples e refletem o impacto da doença, avaliada por meio das respostas global e integrada dos sistemas pulmonar, cardiovascular e muscular periférico (2) a fim de estabelecer um programa de reabilitação pulmonar adequado às limitações e a gravidade da doença.

Em relação aos testes físicos funcionais, destacam-se os testes submáximos (5-7) como formas alternativas aos testes máximos para avaliação da capacidade funcional, por não requererem equipamentos de alto custo, sendo de fácil aplicação na rotina clínica (8-11).

Os testes submáximos, comparados aos testes de cicloergômetro incremental (cardiopulmonar máximo), mostraram-se também mais sensíveis para detecção da dessaturação de oxigênio (queda na saturação periférica de oxigênio: $\Delta \mathrm{SpO}_{2} \geq 4 \%$ ), o que pode fornecer uma informação prognóstica adicional sobre a taxa de mortalidade, tornando-os os melhores testes para a avaliação da necessidade de prescrição de oxigenoterapia $(2,10-13)$.

Considerando que existem diferentes testes funcionais submáximos com o objetivo de avaliar a capacidade funcional dos pacientes com DPOC, como os testes de caminhada de seis minutos em corredor (TC6) (5) e em pista oval (TC6Po), o teste do degrau de seis minutos (TD6) $(6,14)$ e o teste de sentar-se e levantar-se da cadeira em dois minutos (TSL) (7), ainda não está claro na literatura se os mesmos apresentam comportamento da $\mathrm{SpO}_{2}$, sintomas de dispneia e fadiga de MMII semelhantes; tampouco está esclarecido se existe concordância entre o TC6, que é um teste padronizado e validado na literatura científica (5), com os demais testes submáximos ou mesmo quais testes detectam as limitações dos pacientes com DPOC de obstrução moderada a muito grave, justificando assim a realização deste estudo.

Portanto, os objetivos deste estudo foram comparar, em pacientes com DPOC, a $\mathrm{SpO}_{2}$, sintomas de dispneia e fadiga de MMII nos vários testes funcionais citados (TC6, TC6Po, TD6 e TSL); além de verificar se há correlação e concordância em relação à menor $\mathrm{SpO}_{2}$ obtida durante o teste, delta de variação da dispneia ( $\Delta$ dispneia) e delta de variação da fadiga de MMII ( $\Delta$ fadigaMMII) entre o TC6 e os demais testes (TC6Po, TD6 e TSL).

\section{Materiais e métodos}

\section{Sujeitos do estudo}

Foram avaliados inicialmente 16 indivíduos do sexo masculino, na faixa etária de 58 a 81 anos, apresentando DPOC de obstrução moderada a muito grave (15).

De acordo com os critérios de inclusão do estudo, consideraram-se aptos a participar: pacientes do sexo masculino com diagnóstico clínico e espirométrico de DPOC de obstrução moderada a muito grave (relação volume expiratório forçado no primeiro segundo pela capacidade vital forçada $-\mathrm{VEF}_{1} / \mathrm{CVF}-<70 \%$; e $\mathrm{VEF}_{1}$ em valor percentual do previsto $\left(\mathrm{VEF}_{1} \%\right.$ prev) $<80$ (estágios II, III e IV)) (15), confirmado pela espirometria pré e pós-BD (uso de broncodilatadores), realizada sob a supervisão de um pneumologista; que não apresentaram doenças cardíacas, reumáticas e ortopédicas associadas que os impedissem de realizar o protocolo proposto; clinicamente estáveis, sem história de infecções ou exacerbação dos sintomas respiratórios ou mudança de medicamentos há três meses anteriores ao estudo; e sujeitos que não realizaram programa de treinamento físico regular por um período de seis meses anteriores ao estudo; não tabagistas ou ex-tabagistas, e que apresentaram o escore do questionário miniexame do estado mental (16), para avaliação da capacidade cognitiva $>25$. Seriam excluídos os pacientes que passaram a utilizar outro tipo de medicamento durante o estudo, os que apresentaram hipertensão arterial não controlada ou $\mathrm{SpO}_{2}$ abaixo de $80 \%$ ao repouso.

Foram excluídos cinco indivíduos: um por apresentar DPOC de obstrução leve e problemas ortopédicos; um com obstrução moderada que desistiu por problemas pessoais; e três sujeitos com obstrução grave, sendo um devido a intolerância à realização dos testes e dois por apresentarem problemas ortopédicos. A amostra final foi composta por 11 pacientes com DPOC.

Dos 11 pacientes que completaram o estudo, quatro apresentavam DPOC de obstrução moderada (estádio II), cinco tinhamobstruçãograve (estádio III) edoisobstrução muito grave (estádio IV) (15). Todos faziam uso de broncodilatador (BD), sendo que dez associavam-no ao uso 
de corticosteroide inalatório (dose média de $400 \mathrm{mcg}$ ) e um ao uso de corticosteroide oral (dose média de 20 $\mathrm{mg}$ ) sob prescrição médica por pelo menos seis meses anteriores ao estudo. Desses pacientes, somente três eram reagentes ao $\mathrm{BD}$, sendo que nove apresentavam distúrbio ventilatório obstrutivo (DVO) com redução da CVF por hiperinsuflação, um DVO apenas com redução da CVF e um distúrbio ventilatório misto (17).

Todos assinaram o Termo de Consentimento Livre e Esclarecido mediante orientações sobre o protocolo proposto em atendimento à resolução n. 196/96 do Conselho Nacional de Saúde. Este estudo foi aprovado pelo Comitê de Ética da Universidade Federal de São Carlos (UFSCar) (parecer n. 074/2007).

\section{Procedimento Experimental}

Os pacientes foram encaminhados ao Serviço de Fisioterapia Respiratória da UFSCar e submetidos a uma avaliação física geral e específica do sistema respiratório; além do teste espirométrico pré e pós-BD sob responsabilidade de um pneumologista de acordo com as normas da American Thoracic Society/ European Respiratory Society (ATS/ERS) (18).

Testes de avaliação funcional

0 TC6, TC6Po, TD6 e TSL foram realizados em dias consecutivos, com intervalo de no mínimo 24 horas $(2,14)$, randomizados por sorteio e cada teste foi realizado uma vez, entretanto os pacientes foram familiarizados previamente com os testes, sendo todas as avaliações realizadas no período da tarde.

Teste de caminhada de seis

minutos em corredor e em pista oval

0 TC6 foi realizado em corredor plano e coberto com 30 metros de comprimento e 1,5 metros de largura, e o TC6Po foi realizado em uma pista oval com 52 metros de comprimento e 1,5 metros de largura, com demarcações a cada 2 metros, em uma área externa. Os pacientes foram acompanhados pelo avaliador, orientados a caminhar o mais rápido possível durante seis minutos e incentivados com frases preestabelecidas, em intervalos padronizados de um minuto, sendo a cadência dos testes livre.

\section{Teste do degrau de seis minutos}

0 TD6 foi realizado em um degrau de $20 \mathrm{~cm}$ de altura, com piso de borracha antiderrapante (6). Visando melhor funcionalidade, o teste seguiu os mesmos princípios da ATS (5) para o TC6, sendo os pacientes instruídos a subir e descer o degrau o mais rápido possível durante seis minutos, intercalando os MMII, sem o apoio dos membros superiores (MMSS), os quais permaneceram estacionários ao longo do corpo, sendo a cadência de execução livre de acordo com Dal Corso et al. (6).

\section{Teste de sentar-se e levantar-se}

da cadeira em dois minutos

0 TSL foi adaptado e modificado segundo metodologia descrita por Ozalevli et al. (7), e tem sido recomendado como uma alternativa prática para mensurar indiretamente a força dos MMII, considerado um preditor de gravidade (7). Foi utilizada uma cadeira com encosto, sem apoio para os MMSS e altura de $46 \mathrm{~cm}$ (7). 0 teste iniciou-se com o paciente sentado na cadeira, com as costas apoiadas no encosto e os pés apoiados no chão, em seguida, ele foi orientado a sentar completamente na cadeira, se levantar estendendo totalmente os joelhos, sem realizar compensações posturais, mantendo os MMSS cruzados à frente do tórax. Todos os indivíduos deveriam repetir o procedimento o mais rápido possível (7), tantas vezes quanto possível, em um período de dois minutos, sendo a cadência de execução do teste livre.

\section{Medidas de monitorização}

Foram registradas no repouso (basal), no segundo, quarto e sexto minutos do TC6, TC6Po e TD6, e no repouso, primeiro e segundo minutos no TSL, além do primeiro, terceiro e sexto minutos da recuperação em todos os testes, a $\mathrm{SpO}_{2}$ por meio de um oxímetro de pulso portátil $\left(\operatorname{Nonin}^{\circledR}{ }^{\circledR}\right.$, modelo 2500, Minneapolis, Mn, USA), a frequência cardíaca por meio de um frequencímetro de pulso (Polar Vantage NVTM ${ }^{\circledR}$, modelo 1901001, Kempele, Oulu, Finlândia), e os sintomas de dispneia e fadiga de MMII pela escala de Borg modificada CR10 (19). As medidas da pressão arterial, mensuradas com 
o paciente na posição sentada e com um esfigmomanômetro de coluna de mercúrio $\left(\right.$ Oxigel $^{\circledR}{ }^{\circledR}$, São Paulo, SP, Brasil), foram verificadas no repouso, imediatamente após os testes e no primeiro, terceiro e sexto minutos da recuperação.

A diferença da $\mathrm{SpO}_{2}$ basal com a menor $\mathrm{SpO}_{2}$ obtida durante o teste $\left(\Delta \mathrm{SpO}_{2}\right)$ foi considerada indicativo de dessaturação quando maior ou igual a $4 \%$ $(2,12)$. A suplementação de oxigênio seria indicada se a $\mathrm{SpO}_{2}$ fosse menor que $80 \%$ durante os testes associada ao sintoma de dispneia e/ou desconforto respiratório intensos (6). 0 desempenho físico no TC6 e TC6Po foi determinado pela distância total percorrida em metros (DP-TC6 e DP-TC6Po, respectivamente), no TD6 pelo número total de subidas e descidas no degrau (TD6-T) e no TSL pelo número total de repetições completas de sentar e levantar da cadeira (TSL-T).

\section{Análise estatística}

Os resultados deste estudo foram analisados pelo programa Statistica, versão 7.0 (StatSoft, Inc. 19842004, Tulsa, OK, USA). A distribuição dos dados foi verificada pelo teste de normalidade de Shapiro-Wilk. A estatística descritiva foi realizada para a caracterização da amostra e os dados foram expressos em médias \pm desvios padrão e mediana (intervalo interquartílico) para as variáveis de distribuição normal e anormal, respectivamente.

Para a análise intertestes, para comparar o $\Delta \mathrm{SpO}_{2}$ e os deltas de variação ( $\Delta=$ pico - basal) dos sintomas de dispneia e fadiga de MMII, foi utilizado o teste ANOVA de medidas repetidas ou o teste de Friedman, além dos coeficientes de correlação de Pearson ou Spearman, sendo os testes para as variáveis com distribuição normal e anormal, respectivamente. A análise gráfica de concordância entre os menores valores da $\mathrm{SpO}_{2}, \Delta$ dispneia e $\Delta$ fadiga de MMII dos testes funcionais submáximos foi realizada pelo teste de Bland-Altman (20), considerando o TC6 como o teste-padrão, utilizando-se o software MedCalc, versão 12.7.1.0 (MedCalc, Mariakerke, Bélgica).

0 cálculo do poder amostral foi realizado pelo programa GraphPad StatMate 2.0 (San Diego, CA, USA) para Windows, baseado em um estudo piloto no desvio padrão e na diferença das médias do $\Delta \mathrm{SpO}_{2}$, correspondendo a um poder acima de $80 \%$. 0 nível de significância adotado foi de $5 \%$.

\section{Resultados}

As variáveis antropométricas, espirométricas, $\mathrm{SpO}_{2}$ no repouso, desempenho físico no TC6, TC6Po, TD6 e TSL são apresentadas na Tabela 1.0 tratamento medicamentoso manteve-se constante durante o período do estudo. Todos os pacientes completaram com sucesso todos os testes.

\section{Avaliação da capacidade funcional de MMII em diferentes atividades}

Na análise intertestes (Tabela 2), em relação à presença de dessaturação durante o teste $\left(\Delta \mathrm{SpO}_{2} \geq\right.$ $4 \%$ ) (12), $\Delta$ dispneia e $\Delta$ fadiga de MMII, não foram encontradas diferenças significantes entre os testes funcionais realizados. Constatou-se dessaturação em todos os testes submáximos realizados. Da amostra total, seis pacientes apresentaram dessaturação de oxigênio no TC6, sete no TC6Po, seis no TD6 e cinco no TSL. Visto que, um paciente com obstrução moderada e um com obstrução muito grave dessaturaram em todos os testes; dois com obstrução grave dessaturaram no TC6, TC6Po e TD6; um com obstrução grave dessaturou no TC6, TC6Po e TSL; um com obstrução muito grave dessaturou apenas no TD6 e TSL; um com obstrução grave dessaturou no TC6Po, TD6 e TSL; um com obstrução moderada dessaturou apenas no TC6 e TC6Po; e por fim, três pacientes não dessaturaram em nenhum dos testes. Não foi administrada a suplementação de oxigênio durante os testes funcionais submáximos conforme descrito na metodologia (6).

\section{Correlação e concordância da capacidade funcional de MMII em diferentes atividades}

Não foram encontradas correlações estatisticamente significantes entre os valores de $\Delta \mathrm{SpO}_{2}$ com os valores de $\Delta$ dispneia e $\Delta$ fadiga de MMII, tampouco do $\Delta \mathrm{SpO}_{2}$, $\Delta$ dispneia e $\Delta$ fadiga de MMII com a DP-TC6, DP-TC6Po, TD6-T e TSL-T.

Correlações positivas foram observadas entre os menores valores da $\mathrm{SpO}_{2}$ no TC6 comparado aos menores valores da $\mathrm{SpO}_{2}$ nos TC6Po $(\mathrm{r}=0,90 ; \mathrm{p}=$ 0,0001), TD6 ( $r=0,68 ; p=0,032)$, e TSL ( $r=0,49$; $\mathrm{p}=0,12$ ); entre $\Delta$ dispneia e $\Delta$ fadiga de MMII no TC6 
e $\Delta$ dispneia e $\Delta$ fadiga de MMII no TC6Po $(\mathrm{r}=0,75$; $\quad$ e entre o $\Delta$ fadiga de MMII no TC6 com o $\Delta$ fadiga de $\mathrm{p}=0,008$ e $\mathrm{r}=0,88, \mathrm{p}=0,0001$; respectivamente); $\quad$ MMII no TSL $(\mathrm{r}=0,72 ; \mathrm{p}=0,01)$.

Tabela 1 - Características antropométricas, valores espirométricos, $\mathrm{SpO}_{2}$ no repouso, distância percorrida nos TC6 e TC6Po, número de subidas no degrau no TD6 e número de repetições de sentar-se e levantar-se da cadeira no TSL dos pacientes estudados

\begin{tabular}{lc}
\hline Variáveis & Valores (média \pm d.p.) \\
\hline Idade (anos) & $71 \pm 8$ \\
Peso (kg) & $70,0 \pm 9,4$ \\
Altura (cm) & $166,0 \pm 5,0$ \\
IMC (kg/m²) & $25,4 \pm 3,0$ \\
VEF$_{1}(\%$ previsto) & $47,5 \pm 14,7$ \\
CVF (\% previsto) & $76,5 \pm 15,1$ \\
VEF $/$ CVF (\%) & $47,3 \pm 14,2$ \\
VVM (\% previsto) & $51,4 \pm 17,2$ \\
SpO ${ }_{2}$ repouso (\%) & $93 \pm 3$ \\
DP total no TC6 (metros) & $495,5 \pm 88,3$ \\
DP total no TC6Po (metros) & $548,2 \pm 79,3$ \\
Número total de subidas no degrau no TD6 & $90,0 \pm 31,9$ \\
Número total de repetições de sentar-se e levantar-se da cadeira no TSL & $36,4 \pm 7,4$ \\
\hline
\end{tabular}

Legenda: IMC = índice de massa corpórea; $\mathrm{VEF}_{1}=$ volume expiratório forçado no primeiro segundo; $\mathrm{CVF}=$ capacidade vital forçada; $\mathrm{VEF}_{1} /$ $\mathrm{CVF}=$ relação $\mathrm{VEF} / \mathrm{CVF} ; \mathrm{VVM}$ = ventilação voluntária máxima; $\mathrm{SpO}_{2}=$ saturação periférica de oxigênio; $\mathrm{DP}=$ distância percorrida; TC6 = teste de caminhada de seis minutos em corredor; TC6Po = teste de caminhada de seis minutos em pista oval; TD6 = teste do degrau de seis minutos; TSL = teste de sentar-se e levantar-se da cadeira de dois minutos.

Fonte: Dados da pesquisa.

Nota: Os dados foram expressos em média \pm desvio padrão.

Tabela 2 - Valores dos deltas de variação da saturação periférica de oxigênio, sensação de dispneia, fadiga de MMII durante o TC6, TC6Po, TD6 e TSL dos pacientes com DPOC

\begin{tabular}{lccc}
\hline Testes Funcionais & $\mathbf{S S O}_{2}(\%)$ & $\Delta$ Sensação de dispneia & $\Delta$ Fadiga de MMII \\
\hline TC6 & $-4,9 \pm 3,8$ & $1,0(0,5-3,0)$ & $1,0(0-3,0)$ \\
TC6P0 & $-6,2 \pm 5,5$ & $1,0(0,5-2,5)$ & $0,5(0-2,0)$ \\
TD6 & $-4,2 \pm 2,4$ & $2,0(0,5-3,0)$ & $2,0(0,5-2,0)$ \\
TSL & $-3,9 \pm 3,1$ & $0,5(0-2,0)$ & $2,0(0-2,0)$ \\
\hline
\end{tabular}

Legenda: DPOC = Doença Pulmonar Obstrutiva Crônica; MMII = membros inferiores; $\Delta=$ delta de variação; Sp02 = saturação periférica de oxigênio; TC6 = teste de caminhada de seis minutos em corredor; TC6Po = teste de caminhada de seis minutos em pista oval; TD6 = teste do degrau de seis minutos; TSL = teste de sentar-se e levantar-se da cadeira em dois minutos.

Fonte: Dados da pesquisa.

Nota: Os dados foram expressos em média ( \pm desvio padrão) ou mediana (intervalos interquartílicos). 
Como se observa no Gráfico 1, não foi possível constatar concordância entre o TC6 e os demais testes funcionais nas três análises realizadas, visto que, a média da diferença entre as médias com $\pm 1,96$ desvio padrão da menor $\mathrm{SpO}_{2}$ obtida durante os testes, nos pacientes com DP0C, entre TC6 e TC6Po, TC6 e TD6 e entre TC6 e TSL foram de $0,8 \pm 3,5 \% ;-1,9 \pm 3,5 \%$ e $-2,5 \pm 4,6 \%$, respectivamente (Gráfico 1A, 1B e 1C), verificando-se grande variação dos limites de concordância. Em outros termos, a porcentagem de variação da $\mathrm{SpO}_{2}$ obtida nos testes foi muito grande (cerca de $13,2 \%$ para o TC6Po; $13,5 \%$ para o TD6 e 18,1\% para o TSL). Isso demonstra que, de acordo com a $\mathrm{SpO}_{2}$, não se pode concluir que os testes funcionais realizados concordam com o TC6.

Quanto à sensação de dispneia e fadiga de MMII, também não se observou concordância, uma vez que a média da diferença entre as médias com $\pm 1,96$ desvio padrão (limites de concordância inferior e superior) do $\Delta$ dispneia e $\Delta$ fadiga de MMII no TC6 e do $\Delta$ dispneia e $\Delta$ fadiga de MMII no TC6Po foram $0,0 \pm 1,0$ $(-1,9$ a 1,9$)$ e $0,4 \pm 0,8(-1,2$ a 1,9$)$, respectivamente; e do $\Delta$ fadiga de MMII no TC6 com o $\Delta$ fadiga de MMII no TSL foi $0,1 \pm 1,1(-2,1$ a 2,3), constatando-se grandes limites de concordância e grande variação interindividual. Dessa maneira, de acordo com as variáveis citadas, também não se pode concluir que os testes funcionais realizados concordam com o TC6.

\section{Discussão}

Os principais achados deste estudo podem ser assim sumarizados: (a) obtiveram-se respostas perceptuais de esforço e de oxigenação similares entre os testes funcionais, verificada pela comparação $\operatorname{dos} \Delta \mathrm{SpO}_{2}$, $\Delta$ dispneia e $\Delta$ fadiga de MMI; (b) constatou-se dessaturação em todos os testes; (c) percebeu-se correlação, mas não concordância entre os menores valores da $\mathrm{SpO}_{2}$ no TC6 com os menores valores das $\mathrm{SpO}_{2}$ no TC6Po, TD6 e TSL, entre $\Delta$ dispneia e $\Delta$ fadiga de MMII no TC6 com os $\Delta$ dispneia e $\Delta$ fadiga de MMII no TC6Po, e entre $\Delta$ fadiga de MMII no TC6 com o $\Delta$ fadiga de MMII no TSL.

\section{Avaliação da capacidade funcional de MMII em diferentes atividades}

Dos testes funcionais realizados neste estudo, o único que apresenta validação (padronização e implicações clínicas) é o TC6 (5). No entanto, os demais testes são utilizados na avaliação da capacidade de exercício de pacientes com DPOC e são citados na literatura internacional, porém ainda não apresentam validação para a população estudada $(6,7,14)$.

Os resultados deste estudo indicam que não foram encontradas diferenças significantes entre o $\Delta \mathrm{SpO}_{2}$ dos testes submáximos (TC6, TC6Po, TD6 e TSL), sugerindo que as respostas de oxigenação foram semelhantes em magnitude entre os testes e demonstrando demandas similares. Os mesmos resultados apontam que ocorreu dessaturação de oxigênio em todos os testes, confirmando-os como úteis clinicamente e boas alternativas para avaliação da capacidade de exercício desses pacientes $(6,7)$.

A dessaturação deve-se à piora da relação ventilação-perfusão pulmonar, a qual se acentua com o aumento do volume/minuto e da frequência respiratória (21), e às características patofisiológicas da doença (13). Portanto, reflete a maior demanda imposta ao sistema cardiorrespiratório e sugere que nesses testes funcionais a carga de trabalho imposta pode ter sido semelhante, apesar de o TD6 e TSL serem atividades mais localizadas. Outra causa da dessaturação é a maior quantidade de massa muscular ativa na execução da atividade de subir e descer o degrau e de sentar e levantar da cadeira e trabalharem os grupos musculares de MMII contra a ação da gravidade; porém, a certeza dessa afirmação depende da análise, não realizada neste estudo, da demanda metabólica e ventilatória exigida em cada teste.

Corroborando os achados, Dal Corso et al. (6) observaram que o $\Delta \mathrm{SpO}_{2}$ foi similar entre o TD6 e os testes cardiopulmonares máximo e submáximo realizados em pacientes com doença pulmonar intersticial. Os autores concluíram que o TD6 é uma alternativa confiável para estimar o grau de dessaturação e a capacidade de exercício. Em contraponto, Ozalevli et al. (7) constataram que os valores de $\Delta \mathrm{SpO}_{2}$ foram significantemente maiores no TC6 que no teste da cadeira de um minuto em pacientes com DPOC; os autores concluíram, então, que o teste da cadeira pode ser usado como um método alternativo ao TC6 para avaliar a capacidade de exercício, mas não o grau de dessaturação. Todavia, Montes de Oca et al. (14) concluíram que o teste de subir degraus de $16 \mathrm{~cm}$ de altura constitui-se numa opção para avaliar a capacidade funcional máxima dos pacientes com DPOC, enquanto que o TC6 uma opção para avaliar a capacidade de exercício. 
Acredita-se que no TC6 e TC6Po, apesar de serem um tipo de atividade diferente do TD6 e TSL, ocorreu dessaturação semelhante pela diferença mecânica no movimento dos MMSS, ou seja, os músculos dos MMSS são ativados durante a caminhada em alguns pacientes com DPOC, sendo um impulso reflexo para os centros respiratórios, conduzindo à dessincronia toracoabdominal e prejudicando a exalação de gás carbônico (13, 22); ao contrário, no TD6 os MMSS permaneceram estacionários ao longo do corpo e no TSL, os MMSS permaneceram cruzados à frente do tórax.

Percebe-se que a dessaturação de oxigênio no TSL ocorreu somente em cinco pacientes, os quais obtiveram um menor TSL-T. Acredita-se que o tempo de realização de dois minutos do TSL, não foi suficiente para provocar alterações metabólicas, ventilatórias e cardiovasculares e dessaturação de oxigênio, necessitando de um tempo maior que dois minutos para fornecer uma estimativa confiável da capacidade de exercício dos pacientes com DPOC de obstrução moderada a muito grave.

Quanto ao TC6Po, não foram encontrados estudos correlatos na literatura. No entanto, ainda que não significantes, observaram-se maiores valores de dessaturação de oxigênio quando comparado aos demais testes; porém, cabe a ressalva, nesse teste os pacientes caminharam uma distância maior.

No entanto, não foram encontradas correlações estatisticamente significantes entre os valores de $\Delta \mathrm{SpO}_{2}, \Delta$ dispneia e $\Delta$ fadiga de MMII com o desempenho físico nos testes (DP-TC6, DP-TC6Po, TD6-T e TSL-T), concluindo que não se pode afirmar que os pacientes com DPOC que apresentaram maior dessaturação de oxigênio, maior dispneia e fadiga muscular de MMII apresentaram maior diminuição da capacidade de exercício. Corroborando, Poulain et al. (13) e Mak et al. (23) mostraram que o $\Delta \mathrm{SpO}_{2}$ não se correlacionou com a DP-TC6; além disso, Mak et al. (23) também não encontraram correlação do $\Delta \mathrm{SpO}_{2}$ com o grau de dispneia e fadiga muscular. Ao contrário, Rodrigues et al. (24) relataram que a capacidade funcional (DP-TC6) sofre influência da pressão parcial de oxigênio no sangue arterial e é associada a indicadores de fadiga muscular; e Ozalevli et al. (7) observaram correlação negativa significante da DP-TC6 e TSL-T com a dispneia em repouso e em esforço pelo índice de Borg (19), e positiva com a força muscular periférica do quadríceps.

Em relação aos deltas de variação de percepção de esforço, sensação de dispneia e fadiga de MMII, não houve diferença significante entre os testes funcionais realizados; assim, pode-se inferir que as respostas perceptuais foram semelhantes entre os testes, demonstrando demandas perceptuais similares. Tais resultados corroboram com Puente-Maestu et al. (25), os quais observaram que a dispneia piorou de forma não linear nos quatro testes de carga constante realizados em cicloergômetro em taxas de trabalho de alta intensidade (isto é, 65, 75, 85 e 95\% da taxa de trabalho incremental pico). Ao contrário, Dal Corso et al. (6) encontraram maior dispneia e fadiga de MMII no teste cardiopulmonar máximo comparado ao TD6 e teste cardiopulmonar submáximo em pacientes com doença pulmonar intersticial; e Dreher et al. (26) encontraram que o teste de escada resulta em maior sensação de dispneia do que o TC6 em pacientes com DPOC hospitalizados; e Ozalevli et al. (7) realizaram o teste da cadeira de um minuto e o TC6 em pacientes com DPOC e constataram valores de $\Delta$ dispneia maiores no TC6.

Borel et al. (27) encontraram que o desempenho obtido no stepper teste não prediz o desempenho no TC6 em pacientes com DPOC e em jovens saudáveis, porém esse teste avalia a mesma demanda cardiorrespiratória que o TC6. Já Schnaider e Karsten (28) concluíram que o desempenho no TD6 prediz o desempenho no TC6 em pacientes com DPOC exacerbados hospitalizados, e que as respostas cardiorrespiratórias e de percepção de esforço foram equivalentes.

\section{Correlação e concordância da capacidade funcional de MMII em diferentes atividades}

A $\mathrm{SpO}_{2}$ foi escolhida como variável para verificar a concordância entre os testes funcionais submáximos, pelo fato de esta ser considerada uma informação prognóstica adicional da taxa de mortalidade e um parâmetro de avaliação da necessidade de prescrição de oxigenoterapia nos pacientes com DPOC, além de ser uma medida fidedigna $(2,11-13)$. Também por ser mais fácil encontrar um oxímetro de pulso nas clínicas de reabilitação do que um ergoespirômetro, uma vez que, este último é um equipamento complexo e de alto custo. Já a escolha da variável dispneia deve-se ao fato de esta ser considerada um preditor de mortalidade e indicador da gravidade da doença, além de avaliar diferentes aspectos da dispneia nas atividades da vida diária (29). A variável fadiga de MMII foi selecionada 

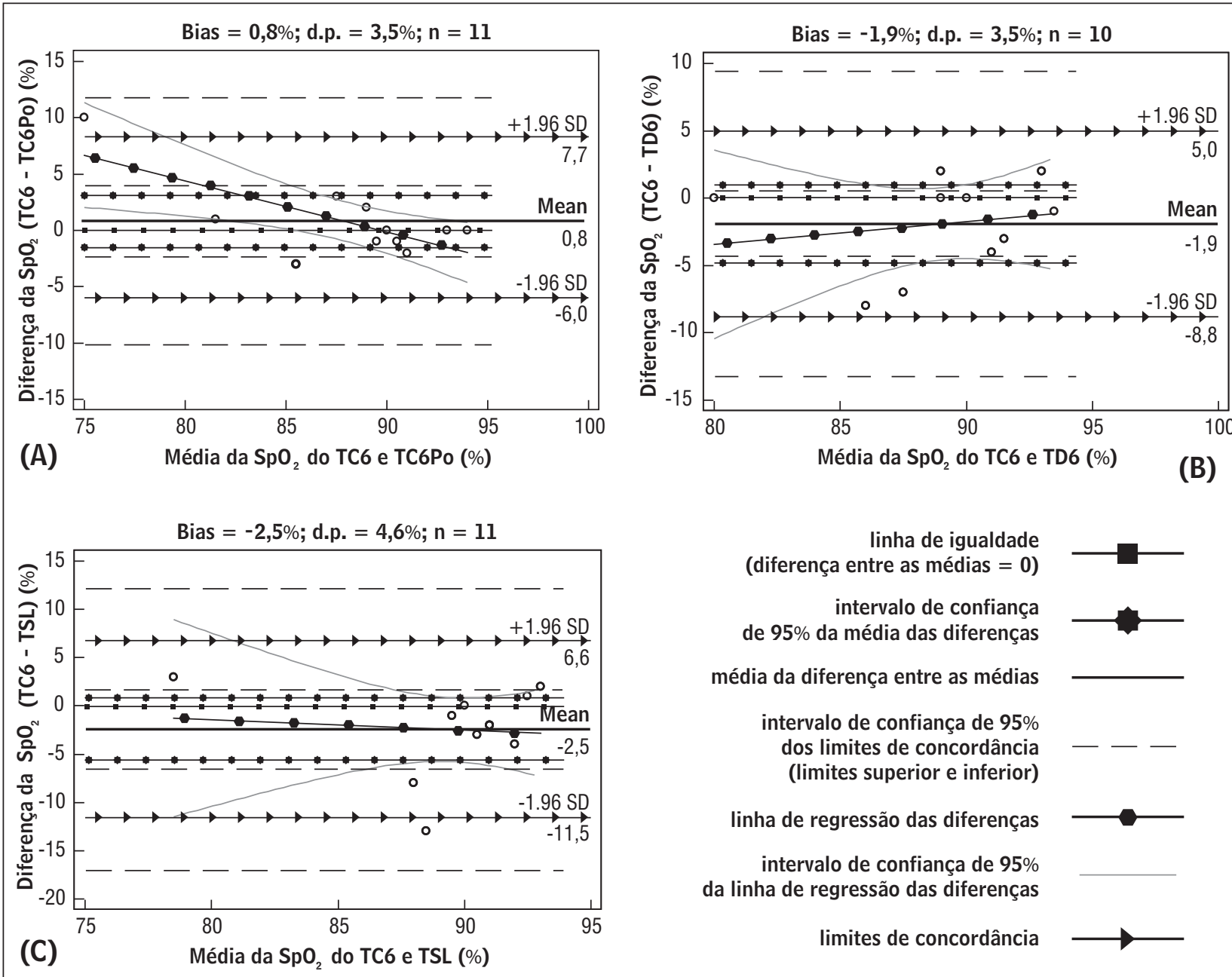

Gráfico 1 - Concordância entre TC6 e TC6Po (A), TC6 e TD6 (B) e TC6 e TSL (C) em relação à menor saturação periférica de oxigênio medida pela oximetria de pulso $\left(\mathrm{SpO}_{2}\right)$ em pacientes com DPOC, de acordo com o teste de Bland-Altman.

Legenda: TC6 = teste de caminhada de seis minutos em corredor; TC6Po = teste de caminhada de seis minutos em pista oval; TD6 = teste do degrau de seis minutos; TSL = teste de sentar-se e levantar-se da cadeira de dois minutos; Bias = média da diferença entre as médias; d.p. = desvio padrão; $\mathrm{n}=$ número de pacientes; $\pm 1,96 \mathrm{SD}=$ intervalo de confiança de $95 \%$.

Fonte: Dados da pesquisa.

porque a hipoxemia conduz a alteração da homeostase da musculatura esquelética periférica, influenciando negativamente a capacidade de exercício nesses pacientes, sendo esta considerada importante marcador da evolução da doença (24). Essas variáveis foram selecionadas por serem facilmente mensuradas pela escala de Borg nos ambientes clínicos (18).

A priori, na literatura científica não foi encontrado nenhum estudo que tenha avaliado e estabelecido um limite de concordância aceitável entre os testes funcionais realizados neste estudo, o que equivale às mudanças clinicamente significantes relacionadas ao comportamento da $\mathrm{SpO}_{2}$ (dessaturação de oxigênio), dispneia e fadiga de MMII durante o esforço físico em testes submáximos. Recomendam-se estudos futuros com o objetivo de avaliar as mudanças clinicamente significantes relacionadas ao treinamento físico aeróbico e descondicionamento físico, estabelecendo previamente uma diferença clinicamente aceitável entre os métodos utilizados.

Não foi possível apurar concordância entre o TC6, considerado teste-padrão, com os diferentes testes funcionais submáximos realizados (TC6Po, TD6, TSL), em relação à menor $\mathrm{SpO}_{2}$ medida pela oximetria de pulso, $\Delta$ dispneia e $\Delta$ fadiga de MMII, de acordo com a visualização da disposição gráfica de 
Bland-Altman (20), visto que apesar da magnitude dos valores de " $r$ " encontrados pelo coeficiente de correlação, observaram-se grandes diferenças dos padrões de distribuição dos escores nos diferentes gráficos (grande variação dos limites de concordância) e grande variação interindividual. Assim, não se pode concluir que as respostas da oxigenação, da dispneia e da fadiga muscular periférica de MMII do TC6 concordam com as do TC6Po, TD6 e TSL, já que o nível de concordância entre eles não seria considerado aceitável para os propósitos clínicos; demonstrando que não é possível avaliar a mesma dimensão das respostas do TC6Po, TD6, TSL a partir do TC6 e que a avaliação deste, não substitui a dos demais testes submáximos. Ressalta-se ainda a sobreposição de valores da $\mathrm{SpO}_{2}$ no Gráfico 1, os quais são representados por 11 pacientes com DPOC.

Devem ser levadas em consideração as variáveis utilizadas para análise de concordância - $\mathrm{SpO}_{2}$, sensação de dispneia e fadiga de MMII — pois talvez se tivéssemos utilizado outras variáveis, como por exemplo, o consumo máximo de oxigênio, poderíamos ter encontrado concordância entre os testes. Dessa maneira, faz-se necessário verificar se os testes podem ser considerados concordantes entre si do ponto de vista da relação do consumo máximo de oxigênio e da sensibilidade às mudanças observadas após um programa de treinamento aeróbico e outras intervenções. Isso porque o TC6 é considerado sensível às mudanças observadas após um programa de reabilitação pulmonar (4), especificamente ao treinamento aeróbico, e também é considerado um preditor de mortalidade na DPOC (30). Dessa maneira, estudos futuros são necessários para avaliar a utilidade clínica dos testes submáximos e a sensibilidade às mudanças proporcionadas pelo treinamento físico na população estudada.

A falta de concordância entre os testes funcionais submáximos realizados no presente estudo não indica a falta de validade e irrelevância clínica do TC6Po, TD6 e TSL na avaliação funcional do paciente com DPOC; apenas compromete o uso intercambiável dos mesmos, ou seja, mostra que a avaliação do TC6Po, TD6 e TSL não substitui a avaliação do TC6. Não há motivo para invalidar nenhum deles, devendo ser considerada aceitável a utilização de qualquer um dos testes realizados neste estudo para avaliar a capacidade de exercício, dependendo da especificidade da reabilitação pulmonar, pois os mesmos indicam diferentes atividades da vida diária, tais como caminhada no solo, atividade de subir e descer escadas e de sentar e levantar da cadeira.

Os testes funcionais submáximos utilizados neste estudo foram escolhidos por serem testes de fácil aplicação e por não requererem equipamentos de alto custo e nem a presença de um médico para sua execução; além disso, ressalta-se que os dados são coletados de forma objetiva e rápida, não necessitando de uma equipe multiprofissional para realização das medidas, embora seja essencial a presença de, no mínimo, um avaliador treinado.

Um aspecto importante no TD6 foi o uso de um degrau simples de $20 \mathrm{~cm}$ de altura, pois degrau com altura inferior poderia fornecer um estresse metabólico insuficiente, o que aumentaria o risco de quedas (6), e degrau com altura superior poderia ser um instrumento difícil para pacientes idosos (6).

\section{Implicações clínicas}

0 TC6 e o TC6Po, considerados testes clínicos de campo, necessitam de um corredor (5) ou de uma pista oval com no mínimo 30 metros de comprimento, dificultando as avaliações de rotina da dessaturação de oxigênio. Ao contrário, o TD6 requer apenas um degrau simples de $20 \mathrm{~cm}$ de altura, podendo ser portátil e realizado em qualquer local, até mesmo em uma sala de atendimento, pois necessita de mínimo espaço físico, e pode ser uma ferramenta adicional e de baixo custo na determinação da capacidade funcional $(6,14)$. 0 TSL, por sua vez, requer apenas uma cadeira de $46 \mathrm{~cm} \mathrm{(7)} \mathrm{de} \mathrm{altura,} \mathrm{a} \mathrm{qual} \mathrm{pode} \mathrm{ser} \mathrm{encontrada}$ até mesmo no domicílio do paciente a ser submetido a um programa de reabilitação. Além disso, em ambos a monitorização é simples e o desempenho físico pode ser facilmente registrado, permitindo avaliar a capacidade de exercício em avaliações de rotina e a dessaturação de oxigênio.

Os resultados do presente estudo podem ter sofrido influência de algumas limitações, tais como: amostra composta unicamente por pacientes do sexo masculino; presença de pacientes com DPOC de obstrução moderada; necessidade de padronização do TD6, do TSL e do TC6, que vêm sendo utilizados para avaliar o desempenho funcional dos pacientes com DPOC; e a não realização de um teste cardiopulmonar submáximo e/ou máximo e assim compará-lo com os testes funcionais realizados, a fim de observar o desempenho dos pacientes. 


\section{Conclusão}

Os testes funcionais submáximos produziram respostas de oxigenação e de percepção de esforço semelhantes em magnitude, avaliando a capacidade de exercício e o grau de dessaturação, nos pacientes com DPOC. Os menores valores da $\mathrm{SpO}_{2}$, $\Delta$ dispneia e $\Delta$ fadiga de MMII do TC6 correlacionaram-se com os dos demais testes (TC6Po, TD6 e TSL), porém de acordo com essas variáveis analisadas não foi encontrada concordância entre eles, pelas grandes variações dos limites de concordância e grande variação interindividual. Dessa maneira, nenhum dos testes substitui o TC6, por serem testes que avaliam e exigem trabalho e uso da musculatura periférica diferentes.

\section{Agradecimentos}

Às fisioterapeutas Vanessa Caravage de Andrade e Ivana Gonçalves Labadessa, à Mestre Cecília Bueno Tesch e à Profa. Dra. Karina Maria Cancelliero, pelo apoio na coleta dos dados.

\section{Referências}

1. Dourado VZ, Tanni SE, Vale AS, Faganello MM, Sanchez FF, Godoy I. Manifestações sistêmicas na doença pulmonar obstrutiva crônica. J Bras Pneumol. 2006; 32(2):161-71.

2. Casanova C, Cote C, Marin JM, Pinto-Plata V, Torres JP, Aguirre-Jaíme A, et al. Distance and oxygen desaturation during the 6-min walk test as predictors of long-term mortality in patients with COPD. Chest. 2008; 134(4):746-52.

3. Killian KJ. Dyspnea: implication for rehabilitation. In: Casaburi R, Petty TL, editors. Principles of pulmonary rehabilitation. Philadelphia: Saunders; 1993. p. 103-13.

4. Kovelis D, Segretti NO, Probst VS, Lareau SC, Brunetto AF, Pitta F. Validação do modified pulmonary functional status and dyspnea questionnaire e da escala do medical research council para o uso em pacientes com doença pulmonar obstrutiva crônica no Brasil. J Bras Pneumol, 2008;34(12):1008-18.

5. American Thoracic Society - ATS. ATS Statement: guidelines for the six-minute walk test. Am J Respir Crit Care Med. 2002;166(1):111-17.
6. Dal Corso S, Duarte SR, Neder JA, Malaguti C, Fuccio MB, Castro Pereira CA, et al. A step test to assess exercise-related oxygen desaturation in interstitial lung disease. Eur Respir J. 2007; 29(2):330-36.

7. Ozalevli S, Ozden A, Itil O, Akkoclu A. Comparison of the sit-to-stand test with $6 \mathrm{~min}$ walk test in patients with chronic obstructive pulmonary disease. Respir Med. 2007; 101(2):286-93.

8. Cataneo DC, Cataneo AJM. Acurácia do teste de escada utilizando o consumo máximo de oxigênio como padrão-ouro. J Bras Pneumol. 2007; 33(2): 128-33.

9. Leung ASY, Chan KK, Sykes K, Chan KS. Reliability, validity, and responsiveness of a 2-min walk test to assess exercise capacity of COPD patients. Chest. 2006; 130(1): 119-25.

10. Casas A, Vilaro J, Rabinovich R, Mayer A, Barberà JA, Rodriguez-Roisin R, et al. Encouraged 6-min walking test indicates maximum sustainable exercise in COPD patients. Chest. 2005;128(1): 55-61.

11. Takigawa N, Tada A, Soda R, Date H, Yamashita M, Endo $\mathrm{S}$, et al. Distance and oxygen desaturation in 6-min walk test predict prognosis in COPD patients. Respir Med. 2007;101(3):561-67.

12. Hadeli KO, Siegel EM, Sherril DL, Beck KC, Enright PL. Predictors of oxygen desaturation during submaximal exercise in 8,000 patients. Chest. 2001;120(1):88-92.

13. Poulain M, Durand F, Palomba B, Ceugniet F, Desplan J, Varray A, et al. 6-Minute walking testing is more sensitive than maximal incremental cycle testing for detecting oxygen desaturation in patients with COPD. Chest. 2003;123(5):1401-7.

14. Montes de Oca M, Ortega Balza M, Lezama J, López JM. Chronic obstructive pulmonary disease: evaluation of exercise tolerance using three different exercise tests. Arch Bronconeumol. 2001;37(2):69-74.

15. Rabe KF, Hurd S, Anzueto A, Barnes PJ, Buist SA, Calverley $\mathrm{P}$, et al. Global strategy for the diagnosis, management, and prevention of chronic obstructive pulmonary disease: GOLD executive summary. Am J Respir Crit Care Med. 2007;176(6):532-55.

16. Bertolucci PH, Brucki SM, Campacci SR, Juliano Y. The mini-mental state examination in a general population: impact of educational status. Arq Neuropsiquiatr. 1994;52(1):1-7. 
17. Pereira CAC, Sato T. Limitação ao fluxo aéreo e capacidade vital reduzida: distúrbio ventilatório obstrutivo ou combinado? J Pneumol. 1991;17(2):59-68.

18. Miller MR, Hankinson J, Brusasco V, Burgos F, Casaburi $\mathrm{R}$, Coates A, et al. ATS/ERS task force: standardisation of lung function testing. Eur Respir J. 2005;26:319-38.

19. Borg GA. Psychophysical bases of perceived exertion. Med Sci Sports Exerc. 1982;14:377-81.

20. Bland JM, Altman DG. Statistical methods for assessing agreement between two methods of clinical measurement. Lancet. 1986;1(8476):307-10.

21. Brown SE, Casciari RJ, Light RW. Arterial oxygen saturation during meals in patients with severe chronic obstructive pulmonary disease. South Med J. 1983;76(2):194-8.

22. Celli BR, Rasullo J, Make BJ. Dyssynchronous breathing during arm but not leg exercise in patients with chronic airflow obstruction. N Engl J Med. 1986;314(23):1485-90.

23. Mak VH, Bugler JR, Roberts CM, Spiro SG. Effect of arterial oxygen desaturation on six-minute walk distance, perceived effort, and perceived breathlessness in patients with airflow limitation. Thorax. 1993;48(1):33-8.

24. Rodrigues SL, Silva CM, Amorim CF, Lima T, Ribeiro FA, Viegas CAA. Correlação entre hipoxemia moderada e função muscular esquelética periférica na doença pulmonar obstrutiva crónica - estudo-piloto. Rev Port Pneumol. 2008;14(6): 769-85.

25. Puente-Maestu L, Pedro JG, Martínez-Abad Y, Oña JMR, Lorente D, Cubillo JM. Dyspnea, ventilatory pattern, and changes in dynamic hyperinflation related to the intensity of constant work rate exercise in COPD. Chest. 2005;128(2):651-56.
26. Dreher M, Walterspacher S, Sonntag F, Prettin S, Kabitz HJ, Windisch W. Exercise in severe COPD: is walking different from stair-climbing? Respir Med. 2008;102(6):912-18.

27. Borel B, Fabre C, Saison S, Bart F, Grosbois JM. An original field evaluation test for chronic obstructive pulmonary disease population: the six-minute stepper test. Clin Rehabil. 2010;24(1):82-93.

28. Schnaider J, Karsten M. Testes de tolerância ao exercício em programa de fisioterapia hospitalar após exacerbação da doença pulmonar obstrutiva crônica. Fisioter Mov. 2006;19(4):119-26.

29. Oga T, Nishimura K, Tsukino M, Hajiro T, Ikeda A, Mishima M. Relationship between different indices of exercise capacity and clinical measures in patients with chronic obstructive pulmonary disease. Heart Lung. 2002;31(5):374-8.

30. Celli BR, Cote CG, Marin JM, Casanova C, Montes de Oca M, Mendez RA, et al. The body-mass index, airflow obstruction, dyspnea, and exercise capacity index in chronic obstructive pulmonary disease. N Engl J Med. 2004;350(10):1005-12.

Recebido: 09/04/2012 Received: 04/09/2012

Aprovado: $13 / 12 / 2012$ Approved: 12/13/2012 